\title{
Distribution of lectin-bindings in the testis of the lesser mouse deer, Tragulus javanicus
}

\begin{abstract}
The distribution of lectin bindings in the testis of the smallest ruminant, lesser mouse deer (Tragulus javanicus), was studied using 12 biotinylated lectins specific for d-galactose (peanut agglutinin PNA, Ricinus communis agglutinin RCA I), N-acetyl-d-galactosamine (Dolichos biflorus agglutinin DBA, Vicia villosa agglutinin VVA, Soybean agglutinin SBA), $\mathrm{N}$-acetyl-d-glucosamine and sialic acid (wheat germ agglutinin WGA, s-WGA), d-mannose and d-glucose (Lens culinaris agglutinin LCA, Pisum sativum agglutinin PSA, Concanavalin A Con A), 1-fucose (Ulex europaeus agglutinin UEA I), and oligosaccharide (Phaseolus vulgaris agglutinin PHA-E) sugar residues. In Golgi-, cap-, and acrosome-phase spermatids, lectin-bindings were found in the acrosome (PNA, RCA I, VVA, SBA, WGA and s-WGA), and in the cytoplasm (PNA, RCA I, VVA, SBA, WGA, LCA, PSA, Con A and PHA-E). sWGA binding was confined to the spermatid acrosome, but other lectins were also observed in spermatocytes. In spermatogonia, VVA, WGA, Con A, and PHA-E bindings were observed. Sertoli cells were intensely stained with DBA and Con A, and weakly with PHA-E. In interstitial Leydig cells, RCA I, DBA, VVA, Con A, PSA, LCA, WGA and PHA-E were positive. UEA I was negative in all cell types including spermatogenic cells. Unusual distribution of lectin-bindings noted in the testis of lesser mouse deer included the limited distribution of s-WGA only in the spermatid acrosome, the distribution of DBA in Sertoli cells, Leydig cells and lamina propria, and the absence of UEA I in all type cells. The present results were discussed in comparison with those of other animals and their possible functional implications.
\end{abstract}

Keyword: Lectin-bindings; Testis; Lesser mouse deer; Tragulus javanicus 